\title{
A renal colic fast track pathway to improve waiting times and outcomes for patients presenting to the emergency department
}

This article was published in the following Dove Press journal:

Open Access Emergency Medicine

24 July 2017

Number of times this article has been viewed

\author{
Omar Al Kadhi' \\ Kate Manley' \\ Madhavi Natarajan' \\ Valmiki Lutchmedial ${ }^{2}$ \\ Abbi Forsyth ${ }^{2}$ \\ Kate Tabrett ${ }^{2}$ \\ Jonathan Betteridge ${ }^{2}$ \\ William Finch $^{3}$ \\ Heinrich Hollis ${ }^{4}$ \\ 'Department of Urology, Norfolk and \\ Norwich University Hospitals NHS \\ Foundation Trust, ${ }^{2}$ Faculty of Medicine \\ and Health Sciences, University of \\ East Anglia, ${ }^{3}$ Department of Urology, \\ ${ }^{4}$ Department of Emergency Medicine, \\ Norfolk and Norwich University \\ Hospitals NHS Foundation Trust, \\ Norwich, UK
}

Correspondence: Kate Manley

Department of Urology, Norfolk and Norwich University Hospitals NHS

Foundation Trust, NNUH, Colney Lane, Norwich, Norfolk NR4 7UY, UK

Tel +4401603286286

Fax +440160328 7657

Email kate.manley@nnuh.nhs.uk
Introduction: Renal colic is commonly encountered in the emergency department (ED). We validated a fast track renal colic (FTRC) initiative to decrease patient waiting times and streamline patient flow.

Method: The FTRC pathway was devised according to the National Institute for Health and Care Excellence clinical summary criteria for the management of patients with suspected renal colic. ED triage nurses use the pathway to identify patients with likely renal colic suitable for fast track to analgesia, investigation and management. Investigations, diagnosis and patient demographics were recorded for 1157 consecutive patients coded as renal colic at a singlecenter ED over 12 months.

Results: Three hundred and two patients were suitable for the FTRC pathway (26.1\%), while 855 were seen by the ED clinicians prior to onward referral. Also, $83.9 \%$ of patients underwent computed tomography scan. In the FTRC group, $57.3 \%$ of patients had radiologically confirmed calculi versus $53.8 \%$ in the non-FTRC group $(p=0.31)$. Alternative diagnoses among FTRC patients $(2.6 \%)$ included ovarian pathology $(n=1)$, diverticulitis $(n=2)$ and incidental renal cell carcinoma ( $n=2)$, while $26.1 \%$ had no identifiable pathology. No immediately life-threatening diagnoses were identified on imaging. Computed tomography scans performed in the non-FTRC group identified two ruptured abdominal aortic aneurysms and alternative diagnoses $(2.57 \%)$ including ovarian pathology $(n=7)$, cholecystitis $(n=2)$, incidental renal cell carcinoma $(n=3)$ and inflammatory bowel disease $(n=1) ; 31.2 \%$ identified no pathology. Time in ED and time to radiologist-reported imaging were lower for the FTRC group versus non-FTRC group $(p<0.0001)$. Conclusion: The FTRC pathway is a safe and efficacious method of reducing diagnostic delay and improving patient flow in the ED.

Keywords: renal colic, ureteric colic, fast track, pathway, patient flow

\section{Introduction}

Renal colic is a common presentation seen in emergency departments (EDs) worldwide. Renal calculi are thought to affect between $7 \%$ and $13 \%$ people, and its incidence appears to be rising in high- and middle-income countries. ${ }^{1}$ With a fairly classical combination of presenting symptoms, this clinical diagnosis is often made in ED and subsequently supported by investigations. Noncontrast computed tomography (NCCT) used to confirm the presence and position of the calculus is the current gold standard imaging modality for this group of patients and is $96.6 \%$ sensitive and $94.9 \%$ specific. ${ }^{2}$

The National Institute for Health and Care Excellence (NICE) clinical knowledge summaries guidance has collated a summary on the best practice for investigation and management of suspected acute renal colic. ${ }^{3}$ A nurse-led fast track pathway was 
developed at our institution based on the NICE guidance for acute management of patients with ureteric or renal colic. The pathway was designed to reduce pressure on ED resources, streamline patient flow through the department, reduce the waiting time for review by a clinician, access to analgesia and for appropriate imaging.

To date, similar pathways involving early use of analgesia and earlier discharge with outpatient investigation have been suggested in the general practice setting to improve patient experience, pain management and reduce the pressure on hospitals with some positive results. ${ }^{4-6}$ Rigorous validation to support their use is limited, however. This study aims to validate and assess the safety of the renal colic fast track pathway by assessing its impact on waiting times, frequency of positive scans and occurrence of nonurologic or life-threatening conditions (such as ruptured abdominal aortic aneurysm).

\section{Methods}

The fast track renal colic (FTRC) pathway was devised according to the NICE clinical summary criteria ${ }^{3}$ (Figure 1). The pathway was designed to direct patients with single kidney, dehydration, signs of shock or sepsis to immediate assessment by an ED clinician, while others with typical features of renal colic were directed to analgesia, fast track NCCT and review by a urology clinician.

Retrospective data were gathered for 1157 consecutive patients coded as "renal colic" following presentation to the ED at a single institution over a 12-month period while the
FTRC pathway was in use. Suitable patients were screened on arrival to the ED by triage nurses using the FTRC protocol; those fulfilling the criteria for the FTRC pathway were given analgesia (per rectum diclofenac if no contraindications or intravenous paracetamol) and sent for further imaging and urology review.

Type of imaging, diagnosis, time until diagnosis and patient demographics were recorded. Statistical analysis was performed using chi-square/unpaired $t$-testing.

\section{Ethical approval}

As per the guidance for research under the National Health Service Health Research Authority in the UK, as the study involves previously collected, nonidentifiable, anonymous patient information, it is excluded from research ethics committee review. The project was registered as a local audit and approved by our institution research and development/ audit office. As the data had already been gathered as part of the electronic records program used in our institution and involved retrospective review of anonymized patient data and was not a clinical trial, it was also exempt from obtaining patient consent in accordance with the National Health Service Health Research Authority Guidance (UK).

\section{Results}

Three hundred and two patients were suitable for and were managed along the FTRC pathway (26.1\%), while 855 were seen by the ED clinicians prior to onward referral - this was

If any entries in shaded boxes, exclude patient from fast track criteria

\begin{tabular}{|l|l|l|}
\hline Exclusion/inclusion criteria & Yes & No \\
\hline Any evidence of hypovolemia or shock? & & \\
\hline Urinalysis positive to leukocytes/nitrites? & & \\
\hline History of pre-existing aortic aneurysm? & & \\
\hline Positive pregnancy test? & & \\
\hline Pyrexia (above $37.8^{\circ} \mathrm{C}$ ) fever, rigors? & & \\
\hline History of abdominal or renal trauma? & & \\
\hline Solitary kidney or kidney transplant? & & \\
\hline Unilateral acute abdominal/back/flank pain? & & \\
\hline Urinalysis positive to blood? & & \\
\hline Patient <60 years old? & & \\
\hline Patient suitable to continue fast track? & & \\
\hline
\end{tabular}

If does not meet criteria, refer to ED clinician for full assessment. If patient $\geq 60$ years old and presents with back, abdominal, and/or flank pain, refer for US or CT abdomen to rule out AAA.

Figure I Renal colic fast track pathway.

Abbreviations: AAA, abdominal aortic aneurysm; CT, computed tomography; ED, emergency department; US, ultrasound. 
due to lack of fulfillment of one or more of the FTRC pathway criteria. Mean average age was not significantly different between the FTRC pathway versus the non-FTRC pathway (45 versus 47 years, $p=0.0816$ ). Also, $83.9 \%$ of patients underwent computed tomography scan. In the FTRC group, $57.3 \%$ of patients had radiologically confirmed renal or ureteric colic, compared to $53.8 \%$ in the non-FTRC group - this was not statistically significantly different ( $p=0.31$ ).

Alternative diagnoses among FTRC patients $(2.6 \%)$ included ovarian pathology $(n=1)$, diverticulitis $(n=2)$ and incidental renal cell carcinoma $(n=2)$, while $26.1 \%$ had no identifiable pathology. No immediately life-threatening diagnoses were identified on imaging.

Computed tomography scans performed in the non-FTRC group identified two ruptured abdominal aortic aneurysms $(0.23 \%)$ and alternative diagnoses $(2.57 \%)$ including ovarian pathology $(n=7)$, cholecystitis $(n=2)$, incidental renal cell carcinoma $(n=3)$ and inflammatory bowel disease $(n=1)$. Also, $31.2 \%$ identified no pathology.

Time to radiologist-reported imaging was lower in the FTRC group versus non-FTRC group (99.9 versus 148.9 $\min , p<0.0001)$.

\section{Conclusion}

Fast track pathways are increasingly utilized in the ED in an effort to improve quality of care and reduce the waiting time to treatment for conditions such as chest pain ${ }^{7}$ or proximal femoral shaft fractures. ${ }^{8}$ This study examines the use of the FTRC pathway for a large patient group and suggests that it is a safe and efficacious method of reducing diagnostic delay and improving patient flow in the ED. NCCT is the gold standard imaging modality for patients presenting with acute-onset flank pain, and the European Urology Guidelines advocate the use of early analgesia in patients presenting with acute colic. ${ }^{2}$ The use of the FTRC pathway facilitates early analgesia and imaging for patients presenting with typical features of renal colic and helps enable appropriate prompt triage of this patient group. There were no life-threatening diagnoses identified in the FTRC pathway group. Time in ED and time to imaging were significantly lower for FTRC patients than for those patients who did not fulfill the FTRC pathway criteria. Multidisciplinary cooperation between the ED and urologic teams is vital for ensuring smooth running of the pathway.

\section{Disclosure}

The authors report no conflicts of interest in this work.

\section{References}

1. Sorokin I, Mamoulakis C, Miyazawa K, Rodgers A, Talati J, Lotan Y. Epidemiology of stone disease across the world. World J Urol. Epub 2017 Feb 16.

2. Turk C, Petrik A, Sarica K, et al. EAU Guidelines on Urolithiasis. Eur Urol. 2015;69(3):475-482.

3. NICE. Renal or ureteric colic - acute. National Institute for Health and Care Excellence - Clinical Knowledge Summaries. 2015. Available from: https://cks.nice.org.uk/renal-or-ureteric-colic-acute\#!scenario.

4. Wright PJ, English PJ, Hungin AP, Marsden SN. Managing acute renal colic across the primary-secondary care interface: a pathway of care based on evidence and consensus. BMJ. 2002;325(7377): $1408-1412$.

5. Xavier A, Maxwell AP. Which patients with renal colic should be referred? Practitioner. 2011;255(1737):15-17, 2.

6. Kastner C, Tagg A. Improving the effectiveness of the emergency management of renal colic in a distinct general hospital: a completed audit cycle. Emerg Med J. 2003;20(5):449-450.

7. Byrne J. Introducing a chest pain pathway in the emergency department to improve quality of care for patients with possible cardiac chest pain. BMJ Qual Improv Rep. 2014;3(1).

8. Ollivere B, Rollins K, Brankin R, Wood M, Brammar TJ, Wimhurst J. Optimising fast track care for proximal femoral fracture patients using modified early warning score. Ann R Coll Surg Engl. 2012;94(4) $267-271$.
Open Access Emergency Medicine

\section{Publish your work in this journal}

The Open Access Emergency Medicine is an international, peerreviewed, open access journal publishing original research, reports, editorials, reviews and commentaries on all aspects of emergency medicine. The manuscript management system is completely online and includes a very quick and fair peer-review system, which is all

\section{Dovepress}

easy to use. Visit http://www.dovepress.com/testimonials.php to read real quotes from published authors. 